\title{
Invasive Lung Adenocarcinoma Mimicking Nodular Tuberculosis in a HIV Positive Patient [Case report]
}

${ }^{1}$ Faculty of Medicine, "Ovidius" University, Constanta, Romania

${ }^{2}$ Clinical Infectious Diseases Hospital of Constanta, Romania

\begin{abstract}
Even though there are many similarities in symptoms and radiological aspect between pulmonary tuberculosis (TB) and lung neoplasia, there are many differences between them like different etiologies, different consequences, and altogether different management. We present a case of a 59 years old male, heterosexual, who was HIV diagnosed in the last 16 years. He had a good immunological and virusological evolution over the time. In the last 5 months of his life he was diagnosed with pulmonary TB and he received specific treatment. After 4 months of antituberculous treatment patient became asthenic, febrile, with productive cough, and weight loss. Imagistic evolution was unfavorable. The suspicion of pulmonary neoplasm raises in the last 3 weeks of his life. Macroscopic lung examination during autopsy was suggestive rather to a pulmonary TB than a lung neoplasm, with a nodular pattern very similar with nodular TB. Histopathological examination evidenced a lung adenocarcinoma.

In HIV patients a delayed or missed diagnosis of lung cancer, can lead to late treatment or wrong treatments, and finally death of patient.
\end{abstract}

Keywords: lung cancer, pulmonary TB, HIV

Danteș Elena

Pneumoftiziology Clinic, Faculty ofMedicine, "Ovidius" University email: elena.dantes@gmail.com tel: +40723472844

\section{Introduction}

The lungs are the most commonly affected organ in patients with HIV either by infectious or by noninfectious pathology.

Lung diseases represent a major complaint in HIV infected persons. Most of the HIV-related morbidity and mortality in HIV infected persons is due to acute and chronic lung disease of infectious or non-infectious etiology. The risk of tuberculosis is far higher than for HIV-uninfected persons and also a particular susceptibility to opportunistic pathogens such as Pneumocystis and Cytomegalovirus; coinfections with other pathogens are also common.

There are a number of common causes of chronic lung disease in HIV infection, such as interstitial pneumonitis and bronchiectasis, that are known to be difficult to differentiate from TB. A rare condition that need to be differentiate from a pulmonary TB is a grossly nodular pattern of a lung adenocarcinoma.

In HIV positive patients from countries with high burden TB diseases, patients with lung cancer are often misdiagnosed as pulmonary tuberculosis, leading to delay in the correct diagnosis as well as exposure to inappropriate medication [1]. 


\section{Material and method}

Retrospective study about hospitalizations and day clinic charts of an HIV positive patient. Pathological autopsy was performed, according to legal provisions. The autopsy specimens were fixed in a solution of $10 \%$ buffered formaldehyde and the sections were embedded in paraffin. Four micrometerthick sections of the lung were prepared and stained with Hematoxylin-Eosin (HE) and Ziehl-Neelsen stains.

Ethic committee agreement from the Clinical Infectious Diseases Hospital Constanta was obtained in order to publish patient data and accompanying images in scientific point of view.

\section{Gase presentation}

We present a case of a 59 years old male, heterosexual, who was HIV diagnosed 16 years ago. He used to be a smoker (over 10 cigarettes/day) for a period of 35 years. He was a compliant patient for HIV treatment point of view, and over the years presented just a few combined antiretroviral treatment (cART) according with therapeutic possibility of each moment, and with side effects to cART (Table I).

Table I - Antiretroviral therapy regimens taken by the patient over the years

\begin{tabular}{|c|c|c|}
\hline No. & cART & Period \\
\hline 1. & $\begin{array}{l}\text { Zidovudine }+ \text { Zalcitabine }+ \\
\text { Efavirenz }\end{array}$ & $\begin{array}{l}\text { November } 1996 \text { - October } \\
1999\end{array}$ \\
\hline 2. & $\begin{array}{l}\text { Zidovudine /Lamivudine + } \\
\text { Efavirenz }\end{array}$ & October 1999 - July 2005 \\
\hline 3. & $\begin{array}{l}\text { Didanosine }+ \text { Stavudine }+ \\
\text { Fosamprenavir/ritonavir }\end{array}$ & July 2005 - August 2009 \\
\hline 4. & $\begin{array}{l}\text { Abacavir/Lamivudine }+ \\
\text { Fosamprenavir/ritonavir }\end{array}$ & August 2009 - February 2011 \\
\hline
\end{tabular}

Good compliance to cART is demonstrated by a favorable immunological and virusological evolution, as it is illustrated in figure no. 1. In the last five months of his life was diagnosed with pulmonary $\mathrm{TB}$ and he received specific treatment. After 4 months of antituberculous treatment patient became asthenic, febrile, with productive cough, and weight lost.

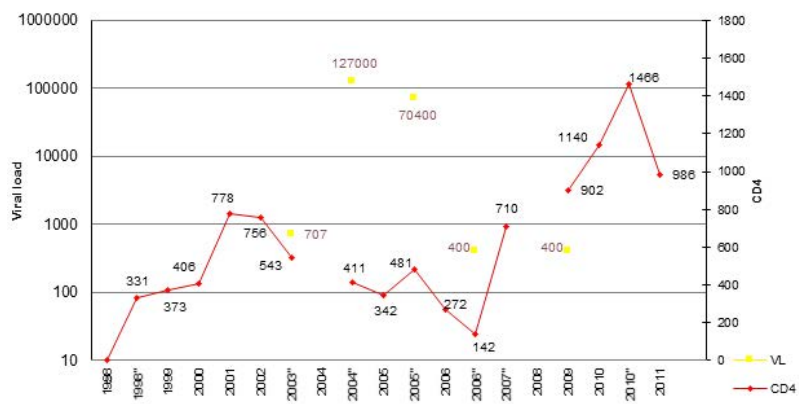

Figure 1 - Immunological and virusological evolution of patient over the years

Since Chest X-ray evolution was unfavorable, we considered necessary to perform a pulmonary CT. CT scan performed in context of worsening clinical evolution rises suspicion of pulmonary neoplasm with three weeks anterior his death (Figure 2).

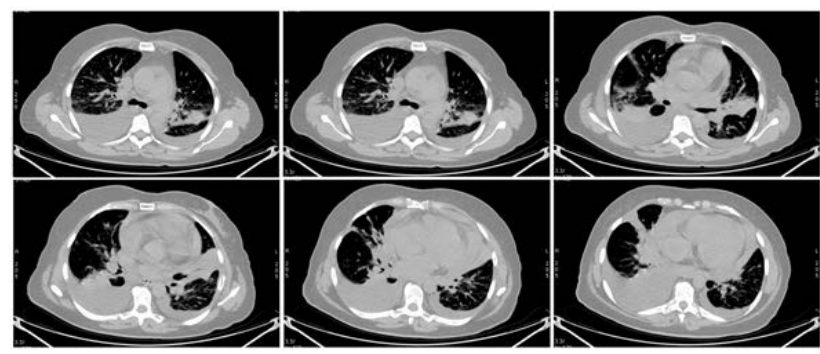

Figure 2 - Pulmonary CT Bilateral multifocal lung lesions associated with mediastinal lymphadenopathy and pleural and pericardial effusion with oncologic character

A pathological autopsy was performed. Macroscopic lung examination was very confusing between pulmonary TB and lung neoplasia, with a nodular pattern very similar with nodular TB as we can see in figure no. $3 \mathrm{~A}$ and $3 \mathrm{~B}$. It were noticed a right pulmonary atelectasis, bilateral massive pleurisy and hilar adenopathy but no macronodular tumoral mass nor metastases grossly visible in other organs were evidenced. 


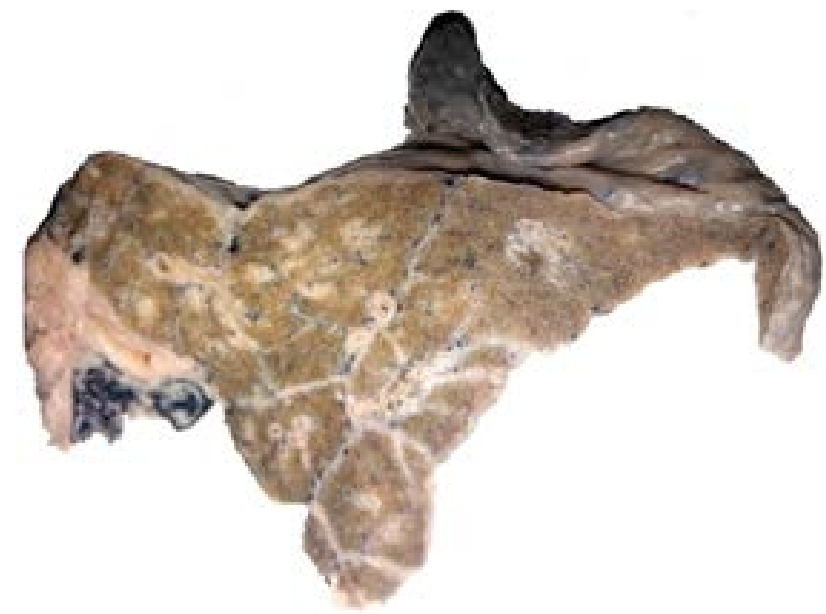

Figure 3A - Transversal section of the lung-macroscopic aspect

Multiple nodular lesions of 0.1-0.2 cm in diameter and thickened wall bronchioles histologically corresponding to atypical adenomatoid hyperplasia lesions and/or lepidic growth pattern of an adenocarcinoma (gross differential diagnosis with miliary TB). Right: lymph nodes with anthracosis and invasion of adenocarcinoma.

Therefore macroscopical autopsy diagnosis pleaded in the first place for nodular tuberculosis.

Histopathological examination evidenced instead an invasive lung adenocarcinoma with lepidic and micropapillary growth pattern shown in figures no. 4A and 4B. Lepidic features showed a proliferation of neoplastic cells along preexisting alveolar structures as we can see in figure no.5. The

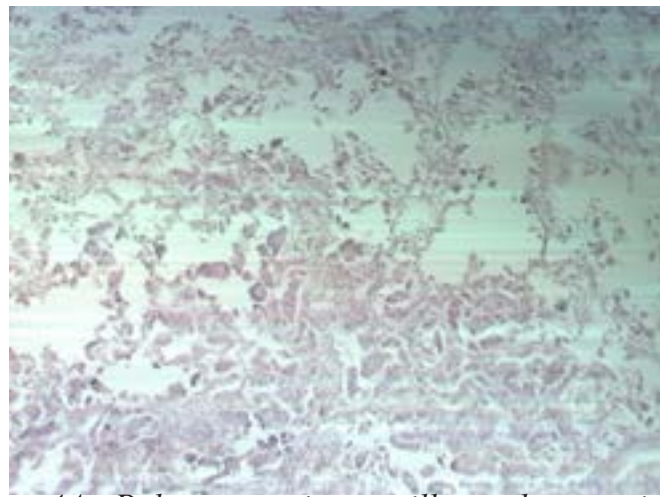

Figure 4A - Pulmonary micropapillary adenocarcinoma

Tumoral cells are arranged in papillary structures (bottom), some of them filling alveolar spaces in the upper field can be identified alveolar spaces. HE stain $x 40$

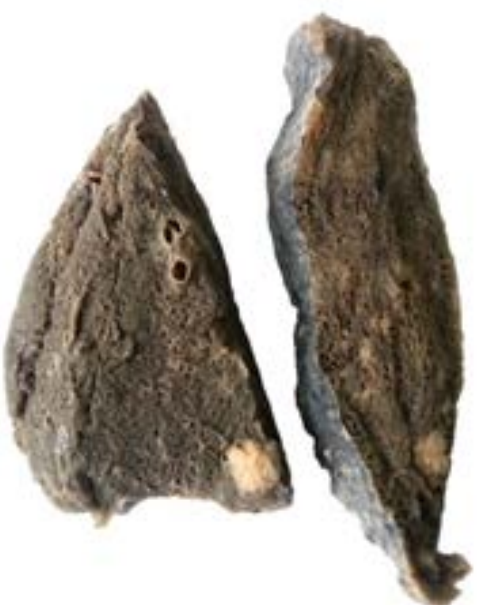

Figure 3B - Longitudinal section of the lung macroscopic aspect

1. Grossly aspect of a nodular lesion of lung, with subpleural and intraparenchymal disposition, relatively well demarcated, of $1 \mathrm{~cm}$ in diameter, tan whitish with necrotic areas. Gross differential diagnosis between nodular TB, metastatic lesion or primitive lung cancer.

2. Lung with small whitish nodular lesions of $0.4 \mathrm{~cm}$ and bronchioles with thickened wall.

micropapillary pattern was highlighted as neoplastic cells growing in papillary tufts lacking fibrovascular cores, aspects illustrated in figure no. 6. Pulmonary hilum lymph nodes evidenced adenocarcinomatous metastases. Malignant cells were observed also on pleural effusions cytology slides.

Ziehl-Neelsen stains evidenced no acid fast bacilli on lung slides or on pleural effusions cytology.

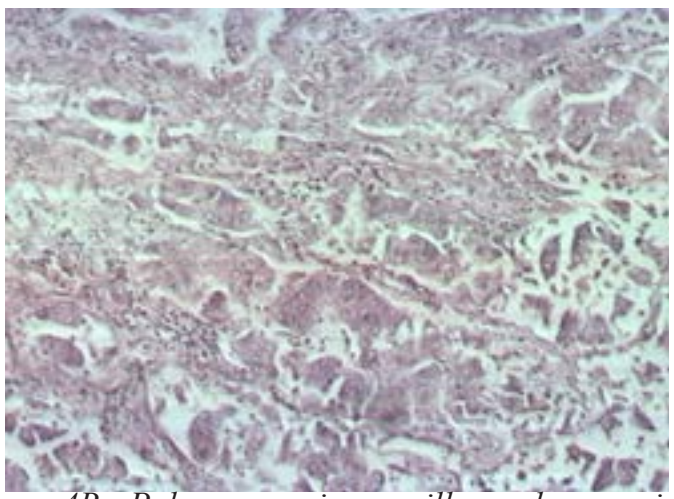

Figure $4 B$ - Pulmonary micropapillary adenocarcinoma

Papillary and micropapillary types of adenocarcinoma consisting of small papillary clusters of glandular cells growing within airspaces, most of which do not show fibrovascular cores. HE stain $x 100$ 


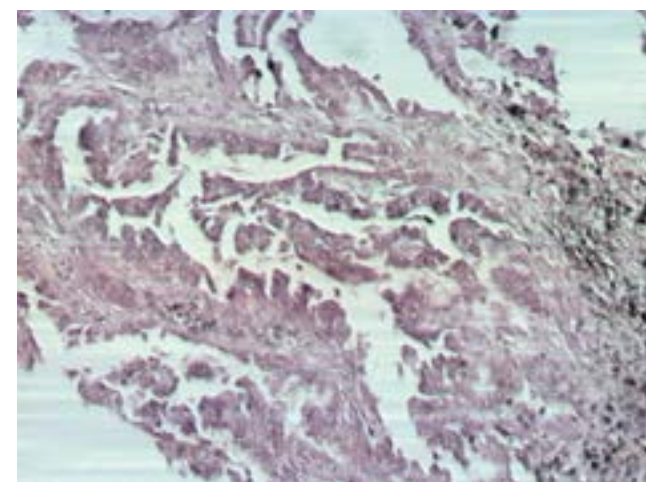

Figure 5 - Lepidic growth of tumoral cells

Lepidic growth of tumoral cells, consisting of their proliferation along the surface alveolar walls and forming also micropapillary structures. Presence of anthracotic pigment deposits in the right. HE stain $x 100$

\section{Discussion}

In Constanta County, according with the study of Chirila S. et al., the incidence of bronchopulmonary cancers, in general population, between years 2007 and 2010 registered an increasing trend with highest value of 5.73 new cases at 10000 inhabitants in year 2010 [2].

Lung cancer is the most common cancer among the non AIDS-defining malignancies in the cART era $[3,4,5]$. Development of lung cancer in patients with HIV has been linked to various factors including immunosuppression, CD4 count, viral load, and smoking [4,5]. HIV infected patients with lung cancer have a more aggressive presentation and a poorer prognosis compared with the general population. Within the HIV population, the incidence of lung cancer is estimated to be approximately 2 to 7 times more than in the general population, fact that can be explained by the high proportion of smokers among HIV-infected persons.

On the other hand pulmonary tuberculosis is one of the most common opportunistic infections in HIV infected persons nonadherent to cART or with late diagnosis of HIV $[1,6]$.

Engels hypothesized that HIV-associated inflammation in the lungs might predispose to

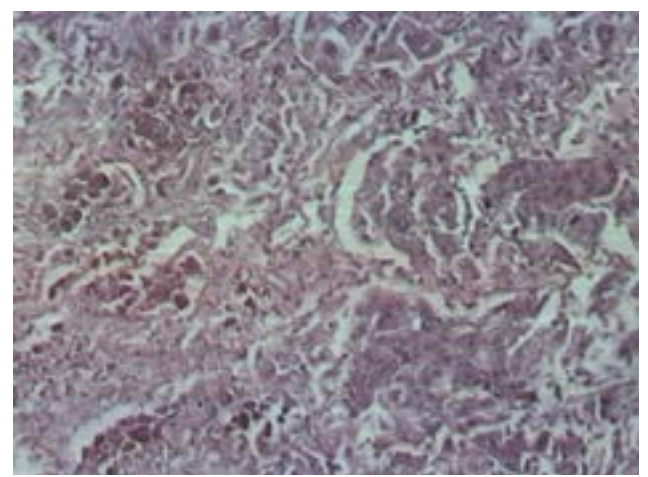

Figure 6 - Micropapillary tumoral proliferation

Malignant tumoral proliferation arranged in micropapillary structures (right); macrophagic alveolitis with siderophages (left). HE stain $\times 100$

smoking-related lung damage [7]. According to Kirk and Shebl, lung cancer in HIV-infected persons has been associated with a history of AIDS-related pulmonary diseases, which are themselves related to immunodeficiency $[8,9]$.

Often these patients presented first time to a physician with advanced disease (stage III or IV) at a younger age and have an inferior overall survival, when compared with non-HIV patients $[4,5]$.

According with study performed by Spano et al the prognosis of lung cancer in HIV positive patients is poor because of their initial extensive disease and a poor response to therapy; the same authors considered that lung cancer is not associated with severe immunodeficiency [10]. In our case, even patients was not too young - almost 60 years old he was diagnosed with extensive disease in context of preserved immunity (CD4 count at the moment of diagnosis was high, around 1000 cells/mmc).

In February 2011, Travis et al. reported, on behalf of multiple multidisciplinary agencies, a revised classification of lung carcinoma, recognizing new subtypes like lepidic and micropapillary (MPP) $[11,12]$.

The micropapillary pattern in lung adenocarcinoma, characterized by papillary structures with epithelial tufts lacking a central fibrovascular core, has been reported to be a new pathological marker of poor prognosis [13].

According with Yoshizawa et al., regarding 
classification of lung adenocarcinoma, lepidic, acinar and papillary adenocarcinomas had an intermediate clinical behavior, with $83-90 \%$ survival at 5 -year, without specific symptoms [12].

It is obvious that evolution of the disease in our case was with at least few years ago, but in context of immunodepression wasn't recognised in due time.

\section{Conclusions}

In this case report, we would like to emphasize that difficulties may be encountered in the differential diagnosis due to the similar symptoms such as asthenia, productive cough and weight loss and risk factors (immunosuppression, smoking) both in tuberculosis and lung carcinoma.

The radiological appearance and grossly aspect may be sometimes confusing in pulmonary $\mathrm{TB}$ and lung adenocarcinoma. In such cases, in which differential diagnosis is difficult, a histopathological examination may help in establishing final diagnosis.

In HIV patients a delayed or missed diagnosis of lung cancer, in a TB endemic area, can lead to late or wrong treatments, with unfavorable clinical outcome and in some situations leading to death of patient.

\section{References}

1. Bhatt, M., Kant, S. \& Bhaskar, R. (2012). Pulmonary tuberculosis as differential diagnosis of lung cancer. South Asian J Cancer. 1, 36-42. DOI: 10.4103/2278-330X.96507

2. Chirilă, S., Rugină, S. \& Broască, V. (2014). Neoplastic Diseases Incidence in Constanta County During 2007 - 2012. ARS Medica Tomitana. 20(4), 211 - 214. DOI: 10.1515/arsm-
2015-0008

3. Ferraresi,A., Calabresi,A., Castelli, F., Vavassori, A., Castelnuovo, F., Scalzini, A., Torti, C. \& Quiros-Roldan, E. (2012). Lung cancer in HIVinfected patients: the experience in Brescia from 1999 to 2009. Int J STD AIDS. 23(10), 753-735. DOI: $10.1258 /$ ijsa.2012.012042

4. Mani, D., Haigentz, M.Jr. \& Aboulafia, D.M. (2012). Lung cancer in HIV Infection. Clin Lung Cancer. 13(1), 6-13. DOI: 10.1016/j. cllc.2011.05.005

5. Pakkala, S. \& Ramalingam, S.S. (2010). Lung Cancer in HIV-Positive Patients. Journal of Thoracic Oncology. 5(11), 1864-1871. DOI: 10.1097/JTO.0b013e3181f387fd

6. Arbune, M. (2013). Characteristic of HIV late presenters in south-eastern Romania. Acta Medica Mediterranea. 29(1), 71- 75

7. Engels, E.A., Brock, M.V., Chen, J., Hooker, C.M., Gillison, M. \& Moore, R.D. (2006). Elevated incidence of lung cancer among HIVinfected individuals. J Clin Oncol. 24, 13831388

8. Kirk, G.D., Merlo, C., O'Driscoll, P., Mehta, S.H., Galai, N., Vlahov, D., Samet, J. \& Engels, E.A. (2007). HIV infection is associated with an increased risk for lung cancer, independent of smoking. Clin Infect Dis. 45, 103-110

9. Shebl, F.M., Engels, E.A., Goedert, J.J. \& Chaturvedi, A.K. (2010). Pulmonary infections and risk of lung cancer among persons with AIDS. J Acquir Immune Defic Syndr. 55, 375379

10. Spano, J.P., Massiani, M.A., Bentata, M., Rixe, O., Friard, S., Bossi, P., Rouges, F., Katlama, C., Breau, J.L., Morere, J.F., Khayat, D. \& Couderc, L.J. (2004). Lung cancer in patients with HIV Infection and review of the literature. Med Oncol. 21(2), 109-115

11. Travis, W.D., Brambilla, E., Noguchi, M., Nicholson, A.G. \& Geisinger, K.R. et al. (2011). International association for the study of lung cancer/american thoracic society/european respiratory society international multidisciplinary classification of lung adenocarcinoma. $J$ Thorac Oncol. 6(2), 244-285. DOI: 10.1097/ JTO.0b013e318206a221 
12. Yoshizawa, A., Motoi, N., Riely, G.J., Sima, C.S., Gerald, W.L., Kris, M.G., Park, B.J., Rusch, J.W. \& Travis, W.D. (2011). Impact of proposed IASLC/ATS/ERS classification of lung adenocarcinoma: prognostic subgroups and implications for further revision of staging based on analysis of 514 stage I cases. Modern Pathology; 24, 653-664. DOI: 10.1038/ modpathol.2010.232

13. Makimoto, Y., Nabeshima, K., Iwasaki, H., Miyoshi, T., Enatsu, S., Shiraishi, T., Iwasaki, A., Shirakusa, T. \& Kikuchi, M. (2005). Micropapillary pattern: a distinct pathological marker to subclassify tumors with a significantly poor prognosis within small peripheral lung adenocarcinoma $(\leq 20 \mathrm{~mm})$ with mixed bronchioloalveolar and invasive subtypes (Noguchi's type C tumors). Histopathology. 46(6), 677-684. DOI: 10.1111/j.13652559.2005.02126.x 